\title{
Space-state robust control of a Buck converter with amorphous core coil and variable load
}

\author{
Rafael Morales , José A. Somolinos , Carlos Morón , Alfonso García
}

\begin{abstract}
A B S T R A C T
Pulse-width modulation is widely used to control electronic converters. One of the most frequently used topologies for high DC voltage/low DC voltage conversion is the Buck converter. These converters are described by a second order system with an LC filter between the switching subsystem and the load. The use of a coil with an amorphous magnetic material core rather than an air core permits the design of smaller converters. If high switching frequencies are used to obtain high quality voltage output, then the value of the auto inductance $L$ is reduced over time. Robust controllers are thus needed if the accuracy of the converter response must be preserved under auto inductance and payload variations. This paper presents a robust controller for a Buck converter based on a state space feedback control system combined with an additional virtual space variable which minimizes the effects of the inductance and load variations when a switching frequency that is not too high is applied. The system exhibits a null steady-state average error response for the entire range of parameter variations. Simulation results and a comparison with a standard PID controller are also presented.
\end{abstract}

\section{Introduction}

Buck converters are widely used for DC high-voltage/DC low-voltage conversion when high efficiency is required. These devices consist of switching systems which are controlled with PWM (Pulse Width Modulation) and an $L-C$ network that is coupled with the load $[1,2]$. Since the parameter values $L, C$, and load $R_{L}$ define the system dynamics, variations in any of them modify the load voltage which is the variable controlled by the converter. Variations to $R_{L}$ are defined by the relation between the output voltage and the current to be drawn from the converter. The capacitance $C$ is considered time invariant and the magnetic induction $L$ is affected by errors in the nominal value when an amorphous core coil is used [3]. These magnetic induction properties could shift over time for a multitude of reasons. These variations may be caused by changes in the coil specifications and/or changes in the properties of the core's magnetic material [4]. These changes in the coil properties may occur for different factors, for instance: variations in the packing factor of coils are usually caused by rapid changes in temperature (heating) which may or may not be permanent [5]. Short circuits between contiguous turns of the coil could appear as a result of any kind of loss of isolation. This may be caused by overheating or abrasion. Inductance variations could be significant depending on the number of turns affected and the total turns.

What is more, in magnetic core coils, it is well known that the induction value decreases over time as a result of changes in the core's magnetic properties such as 
anisotropy direction or coercive field. These variations are produced by material heating or heating-cooling cycles which are similar to undesirable annealing treatments [6-8]. Different authors have demonstrated that the coercive field increases with annealing and the passage of time. This is owing to the growth of the magnetic material's size or to magnetic anisotropy direction dispersion [9-12]. It is also well known that magnetic losses (and therefore the activation energy in magnetic cores) increase with the frequency of the magnetic field $[13,14]$. Finally, temperature and/or ambient conditions may lead to chemical changes in the core $[15,16]$ which are clearly associated with heating. The most relevant ageing effect is related to high frequency current supply leading to the decrement of the induction of the core.

Traditional feedback control systems for $L-C$ circuits use a linear time invariant model with small parameter changes. They exhibit accurate responses when modulation frequencies are much higher than the system frequency [1]. However, a non-nominal design of the control system is required when a modulation frequency that is not too high is selected (in order to limit the heating of the switching elements) and in these operating conditions the parameters of the $L-C$ network cannot be considered constant.

In this work is presented a new state-space control system which is robust to changes in the inductance $L$ and large changes in the load $R_{L}$ (voltage/current) of the converter. If a second order state-space feedback is used, then a null steady-state average error is obtained solely for changes in the inductance $L$. However, large changes in $R_{L}$ cause voltage steady state errors. The addition of a virtual state variable combined with a state-space feedback (producing a third order system) allows accurate responses to be obtained when variations in $L$ and $R_{L}$ are produced in the system. The usability of the proposed state-space control approach for this kind of converters is high since the voltage over the load (or capacitor) and the current across the coil are used as physical space-state variables, the magnitudes can be easily measured, the use of space-state observers is avoided and the computational resources required to implement the controller are few.

The paper is organized as follows: Section 2 is devoted to deriving the dynamic model of the converter. Section 3 presents the control system under nominal and nonnominal conditions. Section 4 analyzes the relative average errors of the converter when non-nominal conditions are included. Section 5 includes numerical simulations illustrating the performance of the proposed approach and a comparison with a standard PID controller. Finally, Section 5 is devoted to the conclusions and suggestions for further work.

\section{Dynamic modeling of the converter}

Fig. 1 illustrates a typical Buck converter with a switch implemented as an $N$ depletion mode MOSFET transistor. The coil $L$ is modeled with an amorphous magnetic core and is represented with two parallel lines [17]. Both the transistor and the diode are considered as ideal components, and their heating is considered only from the point of view of the choice of modulation frequency.

If the current over the coil $i_{L}(t)$ and the voltage across the capacitor $u_{C}(t)$ are used as state variables, $x=\left(\begin{array}{lll}i_{L} & u_{C}\end{array}\right)^{T}$, then the state-space model of the converter is:

$$
\left\{\begin{array}{l}
\dot{x}(t)=A_{C} \cdot x(t)+B_{C} \cdot u(t) \\
y(t)=C_{C} \cdot x(t)
\end{array}\right.
$$

with matrices $A_{C}, B_{C}$ and $C_{C}$ being:

$A_{C}=\left(\begin{array}{cc}0 & -1 / L \\ 1 / C & -1 / R_{L} C\end{array}\right), \quad B_{C}=\left(\begin{array}{c}1 / L \\ 0\end{array}\right), \quad C_{C}=\left(\begin{array}{ll}0 & 1\end{array}\right)$

where $u(t)$ denotes the system input and $y(t)$ represents the converter output $y(t)=u_{R}(t)$ and $u_{C}(t)=u_{R}(t) . L, C$ and $R_{L}$ are the coil autoinductance, the capacity and the load respectively.

If the converter dynamics is represented by its inputoutput relation, then a transfer function between $U(s)$ and $Y(s)$ is obtained, where $s$ is the Laplace complex argument.

$\frac{Y(s)}{U(s)}=G_{C}(s)=\frac{1 / L C}{s^{2}+1 / R_{L} C \cdot s+1 / L C}$

Main nominal values of the converter and its components are indicated in Table 1 . While nominal power is $250 \mathrm{~W}$, a maximum power of $1000 \mathrm{~W}$ can be achieved if maximum output voltage is desired with the same nominal load.

Under these nominal values, $L=L_{0}$ and $R_{L}=R_{L 0}$, the system exhibits a natural frequency of $\omega_{00}=2000 \mathrm{rad} / \mathrm{s}$ and a damped factor of $\zeta_{0}=1$ which correspond to a critically damped system. The system thus fulfils that $L_{0} \cdot C=4 \cdot R_{L 0}^{2} \cdot C^{2}$ and the values are taken from (3) as:

$$
\frac{1}{L_{0} C}=\omega_{00}^{2}=4 \times 10^{6}
$$$$
\zeta_{0}=\frac{1}{R_{L 0} C \cdot 2 \omega_{00}}=1
$$

The use of this input-output representation and the application of the final value theorem signify that the steady-state response of the system (2) with a step input is given by:

$y_{S}=y(t=\infty)=Y(s=0)=U \cdot G_{C}(0)$

The PWM switching frequency was selected to avoid high energy dissipation into the switching element and the amorphous core [17], and to reduce the emission of electromagnetic radiations (EMRs) which are directly related to the increase in the switching frequency. The PWM switching frequency was therefore chosen with a value of $f_{S}=2 \mathrm{kHz}$ which is close to the lower established theoretical limits [18]. The relation between this switching frequency and the nominal system frequency $f_{00}=\omega_{00} /(2 \pi)$ becomes 6.28 , which is close to the lower limit given in [18]:

$5 \leqslant \frac{f_{S}}{f_{00}} \leqslant 40$

The chosen value of the switching frequency allows a very simple dynamic model of the system to be used, in accordance with Eq. (3). A more complex amorphous core 


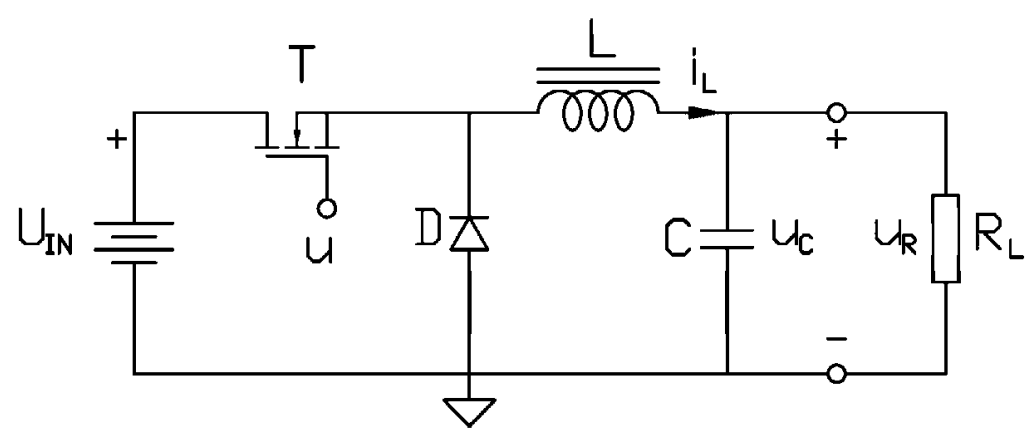

Fig. 1. Buck converter.

Table 1

Main nominal values of the converter.

\begin{tabular}{llrl}
\hline Symbol & Element & Value & Units \\
\hline$U_{I N}$ & DC Source & 100 & $\mathrm{~V}$ \\
$U_{R}=U_{C}$ & Nominal desired output voltage & 50 & $\mathrm{~V}$ \\
$P_{\text {NOM_50 }}$ & Nominal power at $50 \mathrm{~V}$ & 250 & $\mathrm{~W}$ \\
$P_{\text {MAX }}$ & Nominal power at $100 \mathrm{~V}$ & 1000 & $\mathrm{~W}$ \\
$L_{0}$ & Nominal amorphous core coil & 10 & $\mathrm{mH}$ \\
$C$ & Capacity & 25 & $\mu \mathrm{F}$ \\
$R_{L 0}$ & Nominal load & 10 & $\omega$ \\
\hline
\end{tabular}

coil model which integrates the effects of the switching frequency can be neglected. The winding resistance in [17] is not increased at $f_{S}<20 \mathrm{kHz}$ and the main inductance is considered to be frequency independent up to $1 \mathrm{MHz}$. Both limits are quite far from the chosen switching frequency.

Finally, the variation ranges considered for the coil core and the load are the following:

$$
\begin{aligned}
& 0.8 \cdot L_{0} \leqslant L \leqslant L_{0} \\
& R_{L 0} \leqslant R_{L} \leqslant 10 \cdot R_{L 0}
\end{aligned}
$$

In addition, the open circuit regime defined by $R_{L}=\infty$ and short time overloading conditions appearing when $R_{L}<R_{L 0}$ are considered.

Moreover, when an ON-OFF PWM controller is used, the input system $u(t)$ adopts the following form:

$$
u(t)=\left\{\begin{array}{lll}
0 & \text { for } & T k \leqslant t<T(k+1-d(k)) \\
U_{I N} & \text { for } \quad T(k+1-d(k)) \leqslant t<T(k+1)
\end{array}\right.
$$

where $T=\left(f_{S}\right)^{-1}, k$ is an integer, and $0 \leqslant d(k) \leqslant 1$, is the duty ratio (relative pulse width). Steady-state average responses (denoted with subscript $s$ ) for a given average duty ratio, $d_{S}$, then become:

$\bar{y}_{S}=\bar{y}_{S}(t=\infty)=U_{I N} \cdot G_{C}(0) \cdot\left(0+1 \cdot d_{S}\right)$

If expression (9) is normalized for $d_{S}>0$ the following result is yielded:

$\bar{y}_{S_{-} U N I T}(t=\infty)=\frac{U_{I N} \cdot G_{C}(0) \cdot\left(0+1 \cdot d_{S}\right)}{d_{S}}$

Responses from Eqs. (5) and (9) coincide if $U=U_{I N} / d_{S}$ or system (2) includes the scale factor $d_{S}$ in matrix $B_{C}$ (which is similar to scaling the numerator of $G_{C}(s)$ ). These average responses do not depend on the modulation frequency, although their ripple amplitude will be smaller the higher modulation frequency is. The heating of the switching components (transistor $T$ and diode $D$ ) will additionally increase, which is an undesired effect. The addition of switching effects to the whole system produces a faster deterioration in the properties of the magnetic core coil, as was explained in Section 1.

\section{Control system}

The controllability matrix (see [19]) of the given system results in:

$\mathrm{Q}_{C}=\left(\begin{array}{ll}B_{C} & A_{C} \cdot B_{C}\end{array}\right)=\left(\begin{array}{ll}1 / L & 0 \\ 0 & 1 / L C\end{array}\right)$

with range $\left(Q_{C}\right)=2$ for all values of $L, C \neq \infty$. The system is therefore fully controllable.

If $r=r(t)$ denotes the reference signal, then the control input $u$ is composed of a combination of a feed-forward term $K_{W_{0}}$ and a state-space feedback term $K_{F 0}=\left(\begin{array}{ll}k_{10} & k_{20}\end{array}\right)$. This is defined as:

$u=K_{W 0} \cdot r+K_{F 0} \cdot x$

The average scale factor $d_{S}$ is again used, and the continuous control signal $u(t)$ is achieved after the PWM modulator as can be seen in Fig. 2. This figure shows a diagram of this simple state-space control system.

The closed loop system dynamics is governed by the following characteristic polynomial:

$s^{2}+\left(\frac{1}{R_{L} C}-\frac{k_{10}}{L}\right) s+\frac{1}{C}\left(\frac{1}{L}-\frac{k_{20}}{L}\right)-\frac{k_{10}}{L R_{L} C}=0$

The controller gains, which are composed of the feedforward term $K_{W 0}$ and the gain matrix $K_{F 0}=\left(\begin{array}{ll}k_{10} & k_{20}\end{array}\right)$., governing the closed loop dynamics, were set by identifying, term by term, the coefficients of the second degree closed loop characteristic polynomial, with those of the following desired Hurwitz polynomial:

$\phi_{\text {Des }}(s)=s^{2}+\left(\frac{10}{R_{L 0} C}\right) s+\frac{25}{R_{L 0}^{2} C^{2}}$

where the desired eigenvalues of (13), placed in $\sigma_{10}=\sigma_{20}-5 / R_{L 0} C$, were chosen to meet a desirable rising 


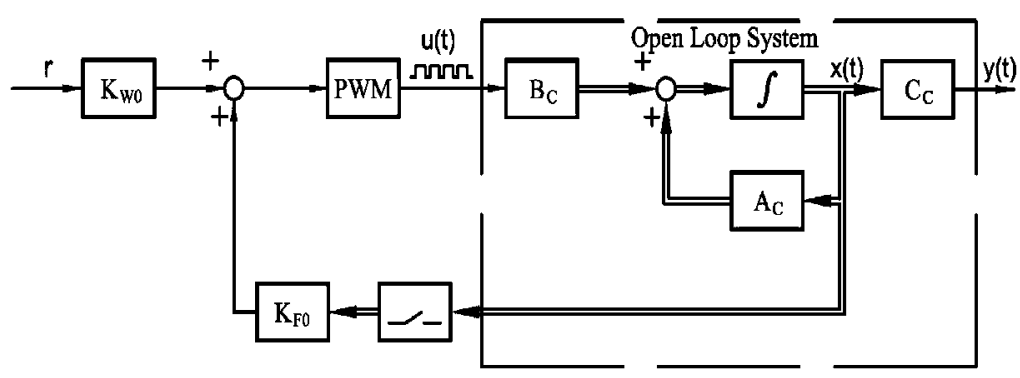

Fig. 2. Nominal state-space feedback

and setting time, five times faster that the original open loop system and without overshooting, implying a critically damped closed loop system. The values of the gain matrix $K_{F 0}$ and the term $K_{w_{0}}$ are therefore given by

$K_{W 0}=100 ; \quad K_{F 0}=\left(\begin{array}{ll}\frac{-9 L_{0}}{R_{L 0} C} & 1-\frac{16 L_{0}}{R_{L 0}^{2} C}\end{array}\right)$

If non-nominal conditions (variations in $L$ and $R_{L}$ ) are considered, then the characteristic polynomial of the controlled system is different from the desired $\phi(s) \neq \phi_{\text {Des }}(s)$ and it becomes:

$$
\begin{aligned}
\phi(\lambda)= & s^{2}+\left(\frac{9 L_{0} R_{L}}{L R_{L 0}}+1\right) \frac{1}{R_{L} C} s \\
& +\left(\frac{9 L_{0} R_{L}}{L R_{L 0}}+\frac{16 L_{0} R_{L}^{2}}{L R_{L 0}^{2}}\right) \frac{1}{R_{L}^{2} C^{2}}
\end{aligned}
$$

Moreover, in order to evaluate the steady-state system response, the relative average error is defined as $\varepsilon_{r}=\left(\bar{r}_{S}-\bar{y}_{S}\right) / \bar{r}_{S}$ where $\bar{r}_{S}$ denotes the average reference value.

According to Eq. (16), if $R_{L}=R_{L 0}$ remains constant and only variations in $L$ are considered, both eigenvalues of the controlled system $\sigma_{1 R_{0}}, \sigma_{2 R_{0}}$ remain real but they vary from the nominal values while the relative average error (from Eqs. (12), (16) and the last term of the denominator of expression (14)) is:

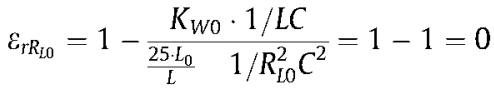

This is not $L$-dependant. However, if non-nominal values in the range given by Eq. (7) of $R_{L}$ are also considered, then new relative eigenvalues change from -23.5 to $-5\left(R_{L 0} C \cdot \sigma_{1}\right)$ and $\left(R_{L 0} C \cdot \sigma_{2}\right)$ from -90.3 to -5 which might imply system frequencies that are higher than $f_{S}$, signifying that the controlled system would consequently be unable to provide acceptable responses. Under these conditions, and according to Eq. (17), the system exhibits a minimum/maximum average relative error which is given by the following expression:

$$
\varepsilon_{r}=1-\frac{25}{9 \cdot R_{L 0} / R_{L}+16}=\left\{\begin{array}{lll}
0 & \text { if } & R_{l}=R_{L 0} \\
-0.479 & \text { if } & R_{L}=10 R_{L 0}
\end{array}\right.
$$

It will be observed that when $R_{L}=\infty$, then the relative error is increased to $\varepsilon_{r}=-0.5625$ and it becomes positive when $R_{L}<R_{L 0}$. From expressions (12) and (13) it will be noted that the control of the system is achieved and the system order is not increased. This is an important advantage of linear state feedback controllers because it is possible to place all the closed loop poles in the desired positions when the system is fully controllable and the numerator of the equivalent closed loop continuous transfer functions does not change with the exception of the feed forward gain. However, it is well known that this solution is unable to respond in an appropriate manner when the system is affected by perturbations. In these cases, the robustness of the system cannot be guaranteed and the linear state feedback controller must be modified, thus increasing the system order, with the inclusion of the output error and by inserting a new "virtual state" or integral action to eliminate it. As a result, the modified system achieves steady-state errors with regard to step inputs, and it additionally improves its degree of perturbation rejection as regards error modeling and external disturbances. This approach is similar to that used in classical control design, in which it is often necessary to add integral actions in order to cancel steady-state errors that track a step input signal (the integral action increases the system type order, but the system order is also increased).

If this approach is particularized to our system, then a new virtual state variable $x_{\varepsilon}$ is created in order to cancel these error sources:

$\dot{x}_{\varepsilon}=r-y=r-C_{C} \cdot x$

and the control signal $u$ (see Fig. 3 ) is now computed as:

$u=K_{\varepsilon 0} \cdot x_{\varepsilon}+K_{F \varepsilon 0} \cdot x$

where the terms $K_{\varepsilon 0}$ and $K_{F \varepsilon 0}=\left(\begin{array}{ll}k_{1 \varepsilon 0} & k_{2 \varepsilon 0}\end{array}\right)$ are the new gain values of the control law designed. The new expanded system is now written as:

$$
\left(\begin{array}{c}
\dot{x}_{\varepsilon}(t) \\
\dot{x}(t)
\end{array}\right)=\left(\begin{array}{cc}
0 & -C_{C} \\
0 & A_{C}
\end{array}\right) \cdot\left(\begin{array}{c}
x_{\varepsilon}(t) \\
x(t)
\end{array}\right)+\left(\begin{array}{c}
0 \\
B_{C}
\end{array}\right) \cdot u+\left(\begin{array}{l}
1 \\
0
\end{array}\right) \cdot r
$$

and using the control law defined in (20), the controlled system becomes:

$\left\{\begin{array}{l}\dot{\hat{x}}(t)=\hat{A}_{C} \cdot x(t)+\hat{B}_{C} \cdot r(t) \\ y(t)=\hat{C}_{C} \cdot \hat{x}(t)\end{array}\right.$

$\hat{A}_{C}=\left(\begin{array}{ccc}0 & 0 & -1 \\ K_{0 \varepsilon} / L & K_{1 \varepsilon 0} / L & \left(K_{2 \varepsilon 0}\right) / L \\ 0 & 1 / C & -1 / R_{L} \cdot C\end{array}\right) \quad \hat{B}_{C}=\left(\begin{array}{l}1 \\ 0 \\ 0\end{array}\right)$ 


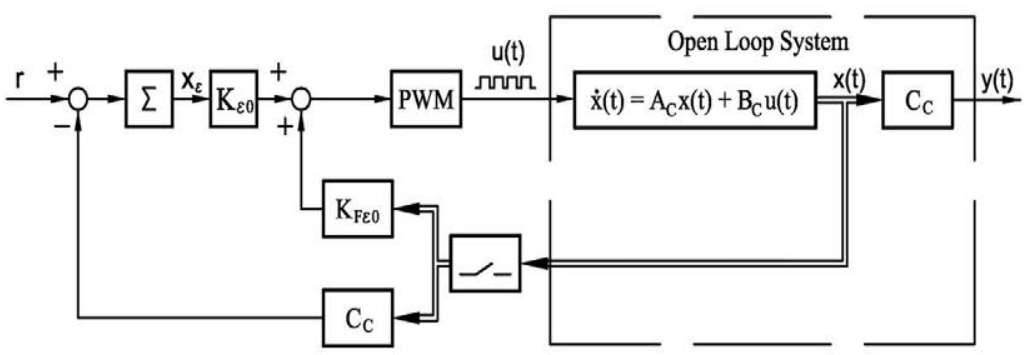

Fig. 3. State-space feedback with a virtual state variable.

Following a similar procedure to that of the previous case, the closed loop characteristic polynomial is achieved from $\phi_{\text {Des }}(s)=\operatorname{det}\left(s \cdot I^{3 \times 3}-\hat{A}_{C}\right)$ as

$$
s^{3}+s^{2}\left(\frac{1}{R_{L} C}-\frac{k_{1 \varepsilon 0}}{L}\right)+s \frac{1}{C}\left(\frac{1-k_{2 \varepsilon 0}}{L}-\frac{k_{1 \varepsilon 0}}{R_{L} L}\right)+\frac{K_{0 \varepsilon}}{L C}=0
$$

The controller gains, composed of $K_{\varepsilon 0}$ and $K_{F \varepsilon 0}=\left(\begin{array}{ll}k_{1 \varepsilon 0} & k_{2 \varepsilon 0}\end{array}\right)$, which govern the closed loop dynamics, were set by identifying, term by term, the coefficients of the third degree closed loop characteristic polynomial, with those of the following desired Hurwitz polynomial:

$\phi_{\text {Des }}(s)=s^{3}+\frac{11}{R_{L 0} C} \cdot s^{2}+\frac{35}{R_{L 0}^{2} C^{2}} \cdot s+\frac{25}{R_{L 0}^{3} \cdot C^{3}}$

where the desired eigenvalues of (24), were placed in the same position to that of the previous case, i.e. $R_{L 0} C \cdot \hat{\sigma}_{1}=R_{L 0} C \cdot \hat{\sigma}_{2}=-5$, and the third eigenvelue, $R_{L 0} C \cdot \hat{\sigma}_{3}=-1$, which acts as the dominant eigenvalue, was placed to exhibit a similar dynamic response than the open loop dynamics, without overshooting from step inputs and to avoid saturation in the PWM actuator. Upon identifying the coefficients in (24) and (25), the following values for $K_{F \varepsilon 0}$ and $K_{\varepsilon 0}$ are thus yielded:

$K_{\varepsilon 0}=\frac{100}{R_{L 0} C} ; \quad K_{F \varepsilon 0}=\left(\begin{array}{ll}-10 L_{0} & -99 \\ R_{L 0} C & 0\end{array}\right.$

\section{Steady state behavior}

In this section the relative average errors are analyzed with the controller defined by Eq. (20). The feedback gains are achieved from Eq. (26) and non-nominal conditions have been included. The characteristic polynomial of the matrix $\hat{A}_{C}$ becomes:

$$
\begin{aligned}
\phi(s)= & s^{3}+\left(\frac{1}{R_{L}}+\frac{10 L_{0}}{R_{L 0} L}\right) \frac{1}{C} s^{2}+\left(100+\frac{10 L_{0}}{R_{L 0} R_{L} C}\right) \frac{1}{L C} S \\
& +\frac{100}{R_{L 0} L C^{2}}
\end{aligned}
$$

It will be observed that expression (27) is different from the desired $\phi_{\text {Des }}(s)$ given in expression (25). From the new last term of this polynomial, the new relative average errors are then cancelled according to:

$\varepsilon_{r \varepsilon}=1-\frac{K_{\varepsilon 0} \cdot 1 / L C}{\frac{100}{R_{\llcorner 0} L C^{2}}}=1-1=0$ for all the values of $L$ and $R_{L}$ contained in bounds defined by (7) in accordance with the relation defined by Eq. (26). Note that the relative error is null even in the cases of $R_{L}=\infty$ and $R_{L}<R_{L 0}$.

\section{Simulation results}

Various simulations have been carried out using the linearization technique described in [20] with $f_{s}=2 \mathrm{kHz}$ and varying the parameters $L$ and $R_{L}$. The proposed controllers were simulated using software tools from Mathworks ${ }^{(2)}$ Inc. These include MATLAB ${ }^{\circledR}$, with Control and Signal Processing Toolboxes and Simulink ${ }^{(8)}$ with SimPowerSystems ${ }^{\mathrm{TM}}$ / Power Electronics/Mosfet library (see [1]). Similar results have been obtained when an IGBT model is used instead of a depletion $\mathrm{N}$-channel Mosfet model.

Fig. 4 depicts some output responses when the control law (12) is used to control the system with different values of $L$ and $R_{L}$. A step signal is used as a reference with $1 \mathrm{~ms}$ of rise time from 0 to $50 \mathrm{~V}$ at $t_{1}=1 \mathrm{~ms}$. It will be noted that the output has reduced its damping factor and that it cannot reach the reference in the so-called worst case $\left(R_{L}\right.$ $=10 \cdot R_{L O}$ and $\left.L=0.8 \cdot L_{0}\right)$. The steady-state average output is $74.72 \mathrm{~V}$ under this condition while the reference is $50 \mathrm{~V}$. Both values allow a relative average error $\varepsilon_{r}=(50-74.72) / 50=-0.494$ to be obtained, which is in accordance with Eq. (18). The bottom of Fig. 4 shows the PWM controller responses for both the nominal and worst cases.

Fig. 5 illustrates the converter responses when the proposed control law (20)-(26) is used to control the voltage outputs with the same reference signal. The PWM responses for nominal and worst cases are also plotted. Note that the controlled system exhibits slower responses than in the previous case. This is because the desired eigenvalue (pole of the closed loop system) $\hat{\sigma}_{3}$ has been chosen as being smaller than $\hat{\sigma}_{10}, \hat{\sigma}_{20}$ and this pole is responsible for the dominant dynamics of the whole system. The steady-state average errors are thus cancelled for every value of $L$ and $R_{L}$ (included the worst case) which agrees with expression (28).

A comparison between a standard PID controller and the proposed control law is carried out in order to show the time responses obtained from both controllers. The PID controller used is expressed as:

$u_{P I D}(t)=k_{P}(r-y)+k_{D} \frac{d}{d t}(r-y)+k_{I} \int_{t_{0}}^{t}(r-y) d \tau$ 


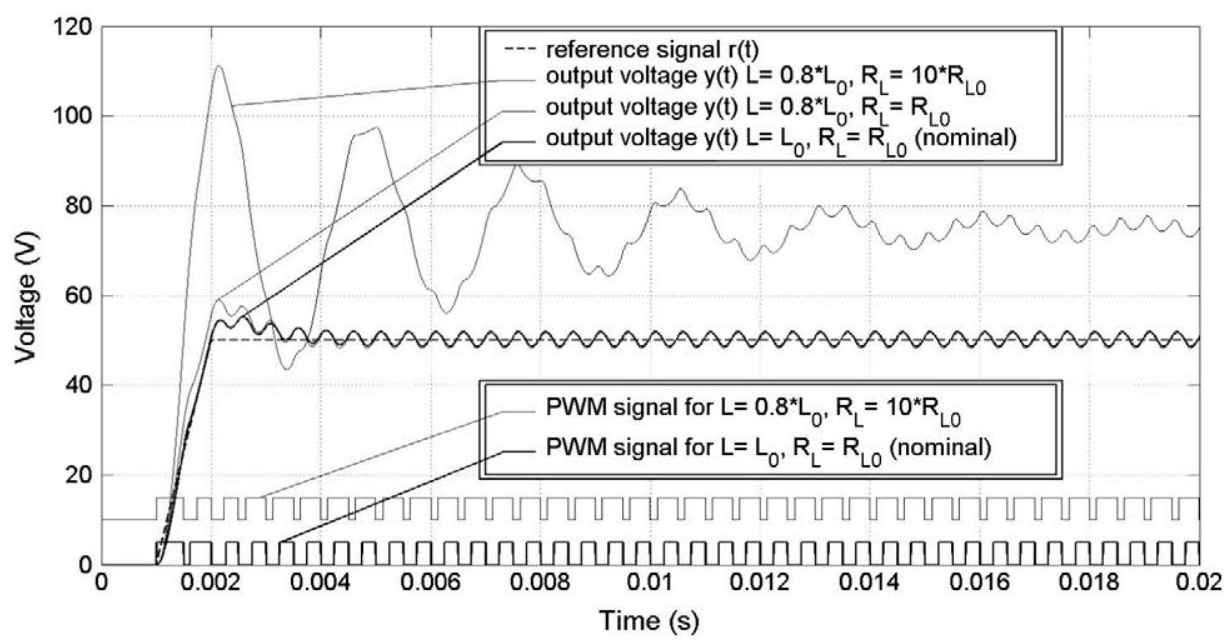

Fig. 4. Time response of the converter under nominal space-state control, inductance aging effect and payload variations.

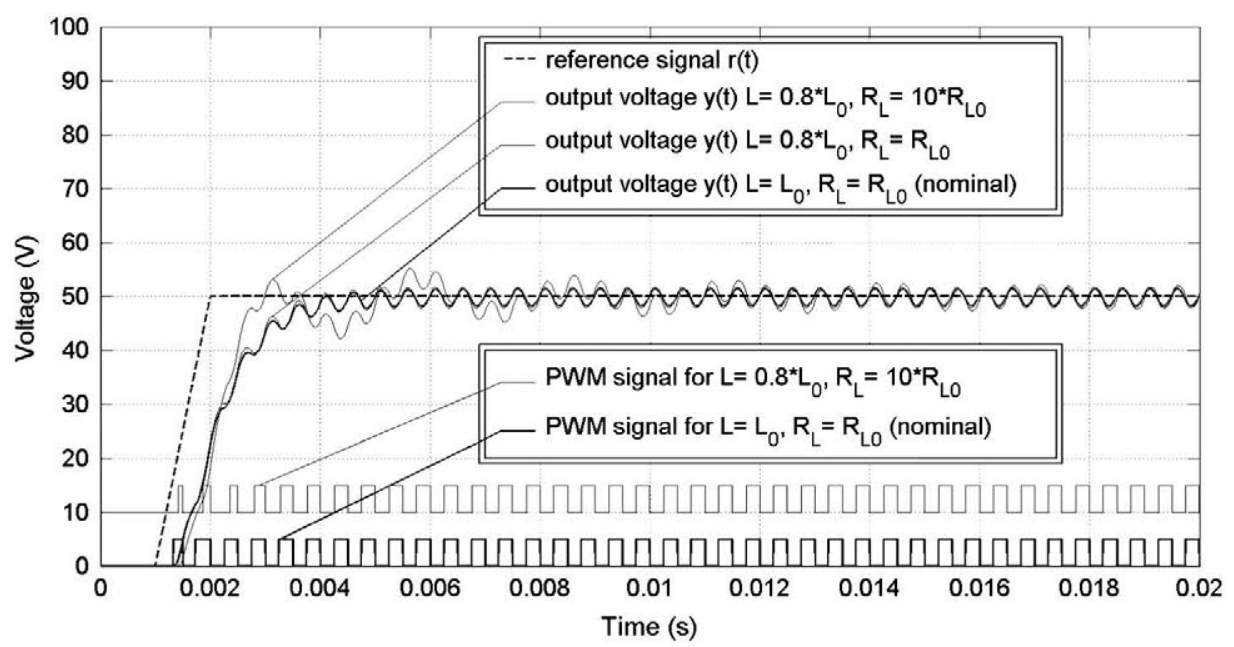

Fig. 5. Time response of the converter under the proposed controller, inductance aging effect and payload variations.

The set of coefficients $\left\{k_{P}, k_{D}, k_{I}\right\}$ were selected to achieve an output voltage control with the same input signal and similar desired eigenvalues for the nominal and the worst case. In these cases, the characteristic polynomial of the controlled system becomes:

$\phi(s)=s^{3}+s^{2}\left(\frac{1}{R_{L} C}+\frac{k_{D}}{L C}\right)+s \frac{1+k_{P}}{L C}+\frac{k_{1}}{L C}$

Upon identifying the coefficients of the PID controller by using direct synthesis from expressions (25) and (30) with nominal values we obtain:

$k_{I}=\frac{100}{R_{L 0} C} ; \quad k_{P}=139 ; \quad k_{D}=\frac{10 L_{0}}{R_{L 0}}$

The equivalent transfer function of the controlled system with the PID controller becomes:

$M_{P I D}(s)=\frac{\left(k_{D} s^{2}+k_{P} s+k_{I}\right) \frac{1}{L C}}{s^{3}+s^{2}\left(\frac{1}{R_{L} C}+\frac{k_{D}}{L C}\right)+s \frac{1+k_{P}}{L C}+\frac{k_{I}}{L C}}$ note that the use of a classical PID controller inserts two zeros which modify the transient response of the system when a non-smooth input signal is used as a reference, owing to their derivative effects. If a modified PID with the derivative part uncoupled from the input is carried out, only a new zero appears in the closed loop equivalent transfer function. The proposed method has the following advantages with regard to the use of typical PID controllers: (a) the controller can be designed in a direct manner (Direct Synthesis) and it is possible to place the system eigenvalues according to the designer criteria; (b) the space-state method uses direct state signal measurements (coil current and capacitor voltage), thus avoiding the use of state-space observers; and (c) the derivative part of the feedback is not coupled with the input and overshooting is avoided when non-smooth reference signals are used thanks to this particular topology.

Fig. 6 depicts the responses of the converter when a PID controller has been implemented. It will be clearly 


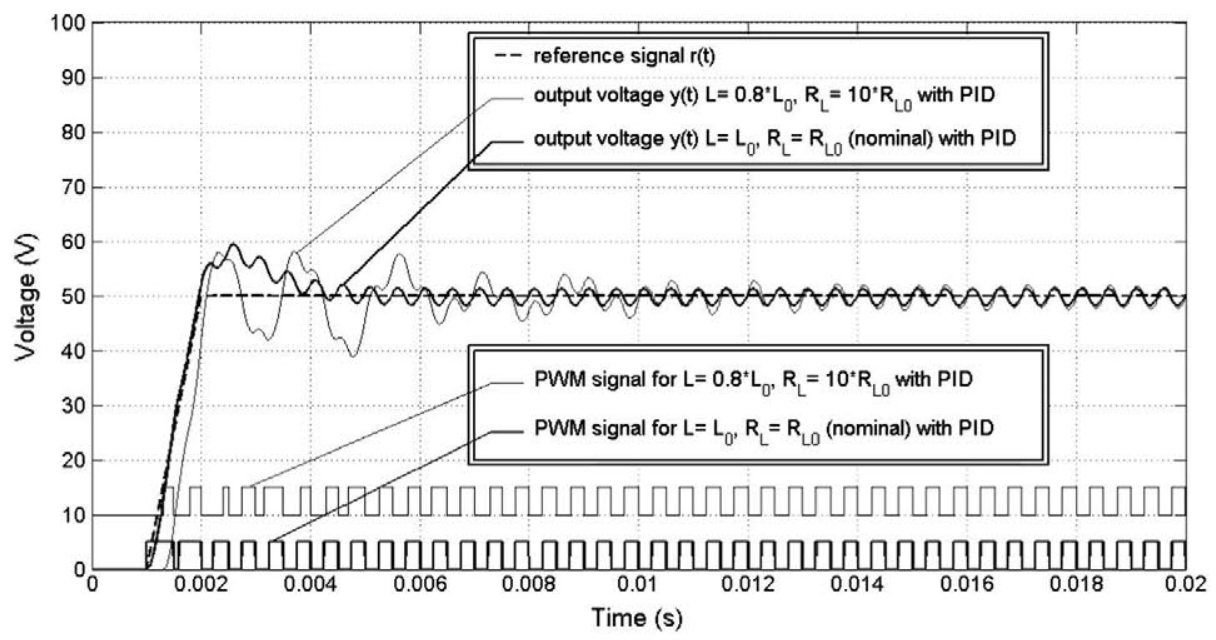

Fig. 6. Time response of the converter with PID controller under nominal and worst-case parameters.

observed how the responses become faster and have undesired overshooting, even under nominal conditions, than the responses obtained from the proposed control law depicted in Fig. 5.

Finally, in order to show the valuable results of the proposed controller, two output responses from two sets of parameters have been plotted in Fig. 7: the nominal response and the previous response used with the considered worst case $\left(L=0.8 \cdot L_{0}\right.$ and $\left.R_{L}=10 \cdot R_{L 0}\right)$ when a new reference signal is applied.

This new reference switches at $t_{1}=1 \mathrm{~ms}$ from $0 \mathrm{~V}$ to $75 \mathrm{~V}$, then drops to $25 \mathrm{~V}$ at $t_{2}=25 \mathrm{~ms}$, and finally reaches the nominal $50 \mathrm{~V}$ at $t_{3}=50 \mathrm{~ms}$. All the transitions are generated with rise/fall times of $1 \mathrm{~ms}$. The relative average errors are cancelled for all the parameter values while the ripple does not increase significantly in either case.

The Mathworks MATLAB ${ }^{\circledR}$-Simulink environment has been used for multiple experiments by authors (see Refs. [21], [22] and [23]). The hardware setup could consist of a Personal Computer with Analog IO isolated interfaces and additional software tools such as Control Toolbox, Real Time Workshop ${ }^{(R)}$ (RTW), Real Time Windows Target ${ }^{(B)}$ (RTWT) and Visual C++ Professional ${ }^{\circledR}$. Since the maximum sampled frequency of the hardware architecture proposed is around $1 \mathrm{kHz}$, it will not be possible to carry out experiments to control power electronics converters despite the relatively small sampling frequency chosen in this work. What is more additional hardware will be required, such as Voltage-PWM converters with additional isolated interfaces, and drivers.

A modular, flexible and reconfigurable hardware setup based on commonly available laboratory devices is therefore proposed. This is based on the Compact Reconfigurable Input-Output architecture (cRIO) from National Instruments ${ }^{(24}$ [2] and the use of LabVIEW ${ }^{(B)}$ which allow the controllers designed to be implemented by using LabVIEW Real-Time ${ }^{(\sqrt{k}}$, LabVIEW FPGA ${ }^{(\mathbb{8})}$ and adequate cRIO Modules. In this case, the cRIO-907x Real Time Controllers

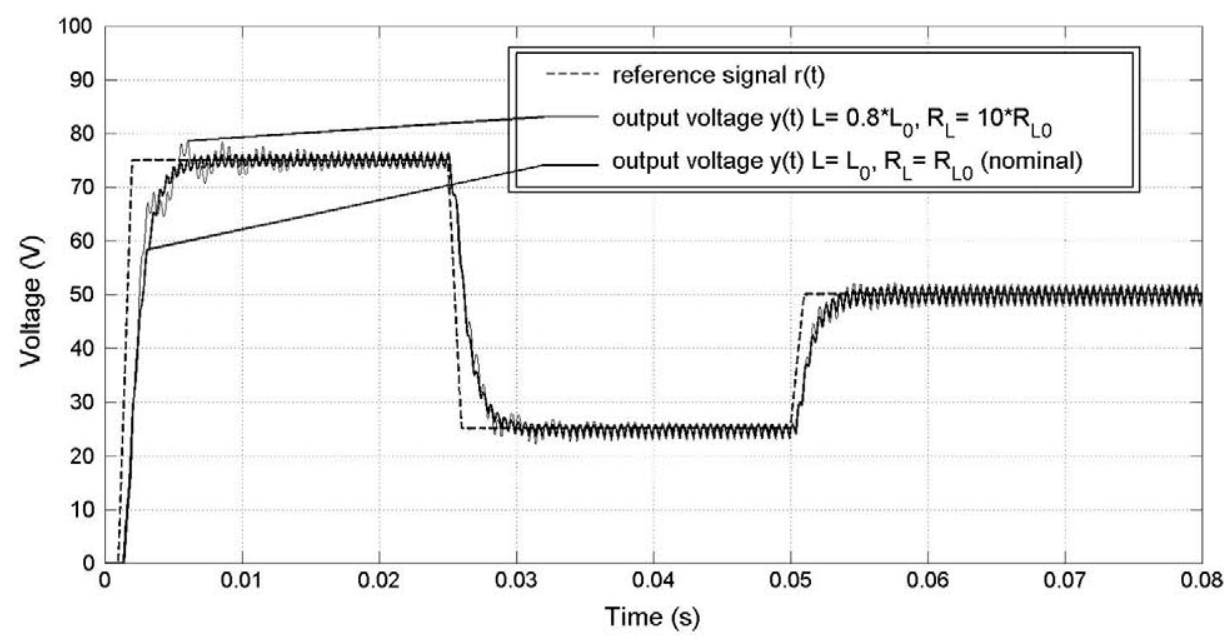

Fig. 7. Output voltage with variable reference for nominal and non-nominal parameters of the converter. 
with a 4 or 8 slot FPGA chassis which provides high $\mathrm{I} / \mathrm{O}$ timing and signal processing performances with switching frequencies of up to $40 \mathrm{MHz}$ can be developed, and the PWM control signals obtained could be directly used to command the switching element with adequate optoisolation. The study of these hardware possibilities needs to be explored and resolved in the future, and they are proposed as topics for further development.

\section{Conclusions}

A robust control of a Buck converter modeled with an amorphous core coil and variable load (voltage and current relation) has been presented. It is controlled via PWM under relatively small modulation frequencies. State-space controllers, which use coil current and capacitor voltage as state variables, can be used to control these converters with fast responses and null steady-state average errors, even in the case of the coil inductance decreasing its value over the time. However, if a non-nominal load is used (which implies that the current drawn from the converter can be very different to the nominal current) the controller response may not operate properly. An additional virtual state variable with integral effect that increases the type order of the system combined with a state-space feedback allows the converter to be controlled over the entire range of parameters, including a decrease in $L$ owing to aging and a high increment of the value of $R_{L}$.

The correspondence between the responses of real electronic circuits and simulation results for these systems is usually very good. A plan to test the simulations has also been introduced to depict how the proposed method can be implemented in the future real platform. Two possible real implementations have been explained based on: (a) Matlab-Simulink-Real Time Workshop-Real Time Windows Target; or (b) CompacRIO-FPGA-LabVIEW. Furthermore, the implementation code is very simple, consisting of the sensor drivers, the designed gains and the integral term, signifying that the implementation of libraries or code-packages is not necessary. Future work will be devoted to verify the effectiveness of the proposed control algorithms and the possible hardware architectures through experiments on a real platform.

\section{Acknowledgments}

The authors would like to thank the anonymous reviewers for their valuable comments and suggestions to improve the quality of the paper.

This work was supported in part by the Spanish Ministerio de Ciencia e Innovación under Grant DPI2011-24113.

\section{References}

[1] N. Mohan, Tore M. Undeland, William P. Robbins, Power Electronics: Converters, Applications, and Design, John Wiley \& Sons, 1997.
[2] P.K. Sadhu, G. Sarkar, A. Rakshit, A microcontroller-based variable voltage variable frequency sinusoidal power source with a novel PWM generation strategy, Measurement 45 (2012) 59-67.

[3] C. Liu, Y. Dong, Resonant enhancement of a passive coil-capacitance loop in eddy current sensing path, Measurement 45 (3) (2012) 622626.

[4] N. Benamrouche, S. Haddad, A. Bousbaine, F.W. Low, Measurement of iron stray load losses in induction motors using the temperaturetime method, Measurement 20 (2) (1997) 91-96.

[5] D. Saidi, B. Zaid, N. Boutarek, N. Bacha, A.S. Ahmed, J.P. Bibérian, Qualitative evaluation of the germination frequency in $\mathrm{Zr}-\mathrm{Fe}$ binary alloy, Measurement 43 (2) (2010) 204-209.

[6] E. Ito, K. Tajima, Y. Kobayashi, Change of the amorphous state by heat treatment below the glass transition temperature, Polymer 24 (7) (1983) 877-882.

[7] B.Y. Fu, D.Y. He, L.D. Zhao, Effect of heat treatment on the microstructure and mechanical properties of Fe-based amorphous coatings, Journal of Alloys and Compounds 480 (2) (2009) 422-427.

[8] M. Belkhaouda, L. Bazzi, A. Benlhachemi, R. Salghi, B. Hammouti, S. Kertit, Effect of the heat treatment on the corrosion behaviour of amorphous $\mathrm{Fe}-\mathrm{Cr}-\mathrm{P}-\mathrm{C}-\mathrm{Si}$ alloy in $0.5 \mathrm{M} \mathrm{H} \mathrm{H}_{2} \mathrm{SO}_{4}$, Applied Surface Science 252 (22) (2006) 7921-7925.

[9] S. Nazlibilek, Y. Ege, O. Kalende, M.G. Sensoy, D. Karacor, M.H. Sazl, Identification of materials with magnetic characteristics by neural networks, Measurement 45 (2012) 734-744.

[10] M.J. Sablik, D. Stegemann, A. Krys, Modeling grain size and dislocation density effects on harmonics of the magnetic induction, Journal of Applied Physics 89 (2001) 7254-7256.

[11] M.F. de Campos, T. Yonamine, M. Fukuhara, F.J.G. Landgraf, C.A. Achete, F.P. Missell, Effect of frequency on the iron losses of $0.5 \%$ and $1.5 \%$ Si nonoriented electrical steels, IEEE Transactions on Magnetics 42 (2006) 2812-2814.

[12] D. Bisero, E. Angeli, F. Spizzo, P. Vavassori, F. Ronconi, Coercive field vs. temperature in Fe/Ag nanogranular films, Journal of Magnetism and Magnetic Materials 262 (2003) 116-119.

[13] A. Lakshman, P.S.V. Subba Rao, K.H. Rao, Frequency dependence of initial permeability and magnetic loss factor of $\mathrm{In} 3+$ and $\mathrm{Cr} 3+$ substituted $\mathrm{Mg}-\mathrm{Mn}$ ferrites, Journal of Magnetism and Magnetic Materials 283 (2-3) (2004) 329-334.

[14] H.G. Brachtendorf, R. Laur, Modeling of hysteresis in magnetic cores with frequency-dependent losses, Journal of Magnetism and Magnetic Materials 183 (1998) 305-312.

[15] G.E. Fish, Stability of magnetic properties of metallic glasses, IEEE Transactions on Magnetics MAG-21 (5) (1985) 1996-2001.

[16] W. McKinnon, J.W. Choung, Z. Xu, J.A. Finch, Magnetic seed in ambient temperature ferrite process applied to acid mine drainage treatment, Environmental Science and Technology 34 (2000) 2576 2581.

[17] M.K. Kazimierczuk, G. Sancineto, G. Grandi, U. Reggiani, A. Massarini, High-frequency small-signal model of ferrite core inductors, IEEE Transactions on Magnetics 35 (5) (1999) 4185-4191.

[18] S. Jung, Y. Tzou, Discrete sliding-mode control of a PWM inverter for sinusoidal output wave form synthesis with optimal sliding curve, IEEE Transactions Power Electronics 11 (1996) 567-577.

[19] K. Ogata, Modern Control Engineering, fifth ed., Pearson, 2010.

[20] Ignacio Nuez, Vicente Feliu, On the Pulse-Width Modulation Control of $L-C$ Filters, IEEE Transactions on Circuits and Systems Part I 47 (3) (2000) 338-349.

[21] R. Morales, H. Sira-Ramírez, Trajectory tracking for the magnetic ball levitation system via exact feed forward linearization and GP control, International Journal of Control 83 (6) (June 2010) 1155 1166.

[22] R. Morales, V. Feliu, H. Sira-Ramírez, Nonlinear control for magnetic levitation systems based on fast online algebraic identification of the input gain, IEEE Transactions on Control Systems Technology 19 (4) (2011) 757-771.

[23] R. Morales, V. Feliu, V. Jaramillo, Position control of very lightweight single-link flexible arms with large payload variations by using disturbance observers, Robotics and Autonomous Systems 60 (2012) $532-547$.

[24] http://www.ni.com/compactrio/. 